\title{
Dynamic Detection on Airflow for Vehicle Intake System Based-on Hot-Film Anemometry Sensors
}

\author{
Rong-Hua Ma, Tsung-Sheng Sheu \\ Department of Mechanical Engineering, ROC Military Academy, Kaohsiung, Taiwan, China \\ E-mail:rh.ma@msa.hinet.net \\ Received November 12, 2010; revised December 17, 2010; accepted December 28, 2010
}

\begin{abstract}
The goal of this study is to develop an airflow meter sensor for vehicle intake system detection in internal combustion engines. The study uses micro-electromechanical process technology to develop a hot-film flow meter with an alumina substrate and platinum film heater; the hotline method is used to create a micro airflow anemometry meter sensor relying on variations in resistance of the platinum film corresponding to different wind velocity at the set temperatures. The micro-sensor is less bulky and simpler structure than ordinary meters, and its small size enables it to provide good sensitivity and measurement precision. The alumina plate used in this study is produced by polishing an alumina substrate, a platinum film is then deposited on the plate to complete the micro-heater used in the sensor. Resistance on the sensor side varies as gas flows through the sensor, and the instrument determines airflow velocity on the basis of the changes in resistance caused by gas flow differences. Airflow velocity form $10 \mathrm{~m} / \mathrm{s}$ to $60 \mathrm{~m} / \mathrm{s}$ are used to test. Resistance displays a regular slope, indicating the relationship between airflow velocities varies remain predictable throughout the sensing range. Therefore, the sensor can achieve its airflow measurement purpose completely.
\end{abstract}

Keywords: Vehicle Intake System, Detection, Anemometry, MEMS

\section{Introduction}

Due to the growth of vehicle density worldwide, plus surging demand for motor vehicles in emerging markets, there will inevitably be stricter vehicle performance requirements in the years to come. In these circumstances, the effective monitoring of engine air intake volume and the resulting energy conversion efficiency will depend crucially on the measurement of air flow rate.

Electronic fuel injection control systems are ubiquitous in motor vehicles, and detective sensors play a vital role in these systems. Although fuel injection has been used on and off for a number of years, the current thrust in emission control and fuel conversation has sparked renewed interest and a number of cars are now being offered with fuel injection systems. Fuel injection can administer a much more closely controlled mixture. While this mixture is not always ideal, it more closely approximates what is required. This permits better gas mileage, smother operation, more power and lowered exhaust emission levels, etc. Taking air flow meters as a typical example, flow meters are used to determine the amount of air taken into the cylinder, enabling an automotive electronic fuel injection system to control fuel injection time on the basis of airflow and engine rpm signals. Apart from being much less bulky than ordinary conventional meters, the small size of micro-sensors made using micro-electromechanical technology enables them to achieve greater precision and sensitivity. Furthermore, micro-electromechanical technology and processes can overcome past mechanical processing obstacles. Due to micro-electromechanical process technology is highly compatible with semiconductor processes, elements can be integrated with semiconductor ICs to form single-chip systems achieving the system miniaturization.

In today's fast-changing technological society, people enjoy many more conveniences and comforts than in the agricultural society of the past. Automation deserves much of the credit for this progress, and the automation that has brought modern convenience depends heavily on sensors. Sensors serve to measure target data, such as temperature, pressure, sound waves, concentrations of specific gases, wind speed, flow velocity, and magnetic 
fields, etc. This data must be processed by the conversion of input signals to output signals, amplification, and integration before it can be interpreted by the system so as to respond correctly and appropriately to external physical phenomena. As a consequence, the ability of sensors to quickly obtain correct data plays a key role in automation, control, and actuation. This is how the need for air stream and airflow direction sensors arose.

Airflow rate and direction sensing are important in many fields, including the monitoring on vehicle engine performance. In past literature on the subject, flow meters were usually classified as thermal and non-thermal sensors on the basis of their sensing method. Among non-thermal gas flow sensors, designs integrating a piezoresistive structure with a cantilever arm are attracting favorable attention due to their high sensitivity, high stability, and good linearity.

\subsection{Micro-Electromechanical Systems}

The physicist Richard P. Feyman first proposed shrinking science and engineering to a microscopic scale at a physics conference in 1959, and suggested that this would be a technological milestone for the future. The invention of the integrated circuit spurred the rapid expansion and development of the electronic element industry. More than forty years later, the electronics industry is developing process technologies capable of achieving even tinier dimensions [1-3]. Apart from enabling many technical improvements, this miniaturization trend has also made electronic elements increasingly inexpensive. Micro-electromechanical system (MEMS) technology is a derivation of semiconductor process technology, and therefore draws on the advantages of semiconductor manufacturing technology [4,5]. MEMS technologies are used in an extremely wide range of interdisciplinary applications spanning the areas of physics, chemistry, electronics, machinery, optics, and materials science, etc. MEMS also represents an improvement on conventional mechanical processing technologies, and offers the advantages of small dimensions, high accuracy, large-scale batch production, and low costs. Furthermore, MEMS technology allows the integration of micro-sensors with other circuits or sensors in order to achieve more powerful functions and greater stability and reliability [6,7]. Adamec et al. [8] fabricated a multi-axis hotwire anemometer with four thermo resistor to evaluate flow direction with a power consumption of $25 \mathrm{~mW}$. Makinwa et al. [9] presented a circular-type thermal flow sensor consists of a heater to detect flow direction and velocity. Recently, non-thermal gas flow meters have been developed. These devices have the advantages of lower power consumption and an improved potential for integration with other sensors. In spite the previous studies investigated many thermal and non-thermal types of flow sensors, those based on hot-films have not been well discussed.

\section{Design}

The flow sensors of the study are fabricated on alumina and utilize platinum resistors as heating and sensing devices. The sensors tested with different micro-heater and sensing mechanism orientations, and investigated the sensing characteristics of single-chip and double-chip sensors of different sizes. The micro-gas sensors investigated in this study employed an alumina substrate on which platinum was deposited to produce a micro-heater and sensing mechanism (Figure 2). Because micro-heaters can continuously produce a controllable, constant temperature environment, providing a stabile power system to the micro-heater can maintain the sensor's operating temperature at the desired level. Wind tunnel experiments were used in conjunction with a connected sensor to observe the amount of temperature varies.

\subsection{Hotline Airflow Anemometry Meter Design Principles}

Hot-line type anemometry meters include several commonly-seen structures: heater, heat sensor, and temperature compensation resistor. Hotline meters employ three chief sensing principles: constant current, constant temperature, and constant power.

1) When there is a constant current, temperature differences caused by the fluid generate different signals.

2) When there is a constant heater temperature, differences in output power can be used to determine the flow rate.

3) When there is constant power, information concerning the fluid can be determined from the effect of the fluid on resistance and current.

The hotline wind speed meter proposed in this study uses a platinum heating resistor that generates heat when a current is flowing. Forced convection will remove the heat, changing the resistance of the heat sensor. In a normal environment, the problem of heat dissipation must be overcome. In Figure 3, the sum of natural convection $Q N$, heat conduction $Q C$, thermal radiation $Q R$, and forced convection $Q F$ is the total heat output of the heater (see Equation (1)). A temperature compensation mechanism can be used to deal with the problems of natural convection and thermal radiation and maintain a balance with the environmental temperature.

$$
P=Q N+Q F+Q C+Q R
$$


Because the hotline anemometry meters are prone to detect errors when the environmental temperature varies, a temperature compensation mechanism must be used. Since temperature and resistance will have a linear relationship within a certain range, appropriate calculations can be used to correct the data in line with the environmental temperature. Because the temperature compensation resistance of a meter is the product of the same processes, it must be very close to the resistance of the meter itself. Equation (2) shows the general relationship between resistance and temperature:

$$
\alpha=R(T-T c) / R_{T}-R_{C}
$$

$R_{T}:$ resistance at temperature $T$

$R_{C}$ : resistance at temperature $T_{C}$

$\alpha$ : resistance-temperature coefficient

\subsection{Design of Hotline Airflow Anemometry Meter Dimensions}

Engine loading is transmitted to the electronic control unit by means of intake manifold sensors signals. The anemometry meter sensor is located in electronic control unit which a protected area away from excessive heat and is connected to the system by means of a wiring harness plug. This hotline airflow anemometry meter has a rectangular shape (see Figures 1 and 2). The gray portion consists of the platinum electrodes; all dimensions are in $\mathrm{mm}$. The double chip has dimensions of $2 \mathrm{~mm} \times 6 \mathrm{~mm}$, and the single chip has dimensions of $6 \mathrm{~mm}$ $\times 6 \mathrm{~mm}$.

\section{Fabrication and Experiments}

Figure 4 shows the chief process steps in the production of the airflow anemometry meter proposed in this study. The alumina substrate was first sent to a wafer fab for polishing (to reduce surface roughness) and cutting to the desired size $(50 \mathrm{~mm} \times 50 \mathrm{~mm})$. Before electron beam evaporation, microlithography was employed to delineate the platinum portions. A spin coater was used to apply the photoresist on the substrate. HMDS was first applied for $30 \mathrm{sec}$. at 3,500 rpm as an adhesive layer, and the substrate was dried for $1 \mathrm{~min}$. at $110^{\circ} \mathrm{C}$ before application of photoresist using the same parameters. The photoresist soft bake required a constant temperature of $110^{\circ} \mathrm{C}$ for $3 \mathrm{~min}$. Exposure was performed after the completion of coating. After confirming no defects, a developer consisting of AZ-400K developer mixed with water in a 1:3 ratio was used to perform development. A diffusion pump and booster pump were used to evacuate the

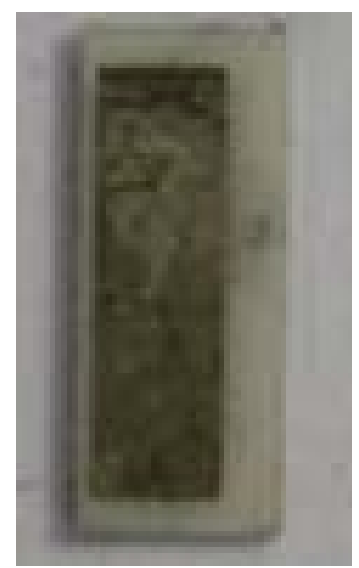

Figure 1. Double chip mask pattern $(2 \mathrm{~mm} \times 6 \mathrm{~mm})$.

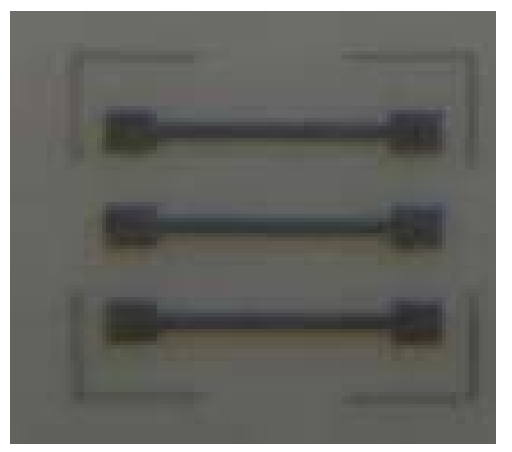

Figure 2. Physical drawing of single chip $(6 \mathrm{~mm} \times 6 \mathrm{~mm})$.

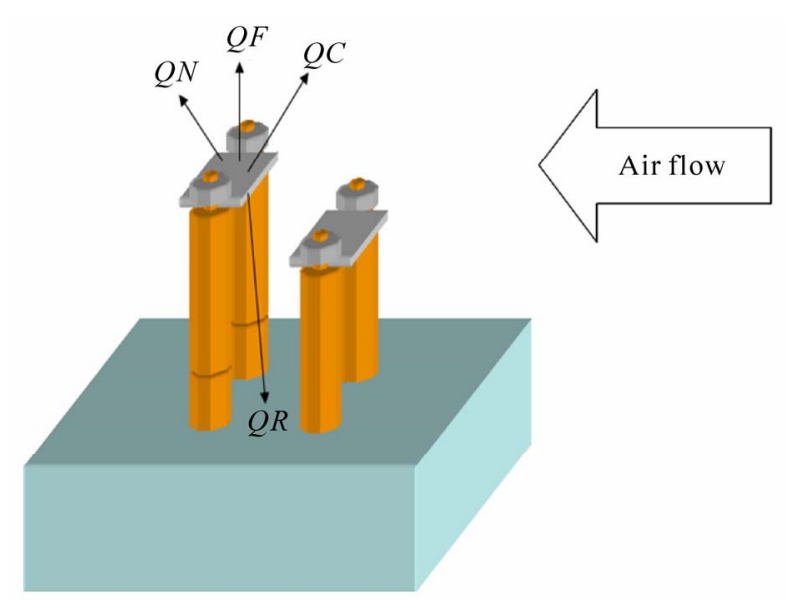

Figure 3. Principle of hotline wind speed meter operation.

vacuum chamber to a background pressure of $2 \times 10^{-6}$ Torr. Before deposition of the platinum, a layer of chromium with a thickness of $0.02 \mu \mathrm{m}$ was deposited on the alumina substrate as an adhesive layer, and electron beam (E-beam) evaporation was used to deposit a platinum layer $0.1 \mu \mathrm{m}$ in thickness. The lift-off method was used to produce parallel electrodes and micro-heaters in the 


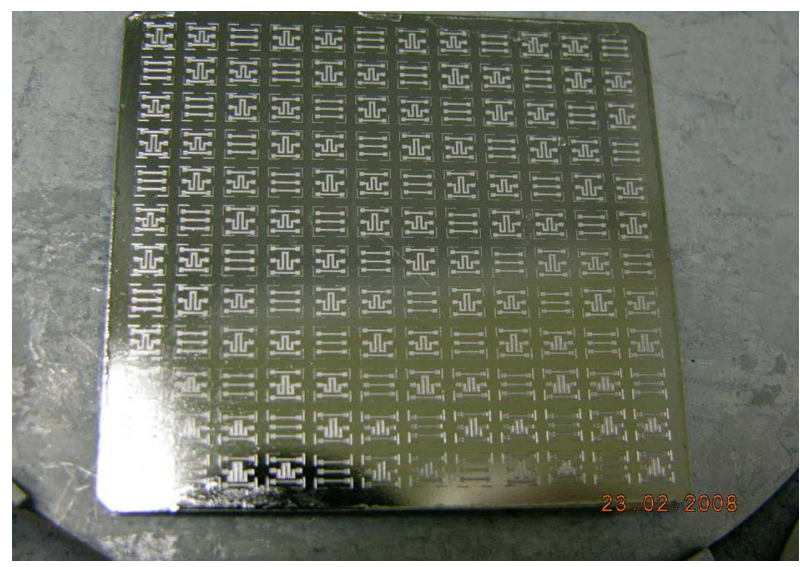

(a)

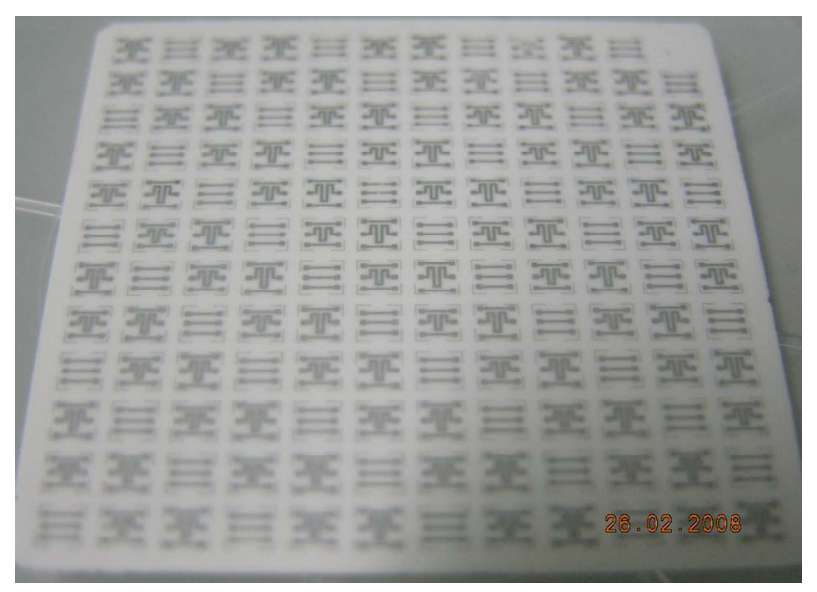

(b)

Figure 4. During lift-off process: (a) Before lift-off; (b) After lift-off.

shape of the pattern (Figure 4). The double chip's micro-heaters had resistance values of $20 \Omega, 30 \Omega$, and 40 $\Omega$, and the single chip's micro-heaters had resistance values of 40-80-40 $\Omega, 80-80-80 \Omega$, and 40-120-40 $\Omega$. It can be seen from Figure 5 that the fabrication process involved the deposition of platinum micro-heaters on an alumina substrate.

\section{Results and Discussion}

This study performed wind speed testing using single and double chips separately. The double chips were mounted on a flat surface, and were oriented with either front sensing and rear heating or front heating and rear sensing (Figure 6). Testing was performed using chips with different resistances in different environmental temperatures to compare the chips and perform optimization (Figures 7-10). As shown, the resistance signals of the increases approximately linearly with increasing airflow velocity, thus confirming the stability of the sen- (a) Spinning on photoresist

(b) Exposure and developing

(c) Electron-beam evaporation of $\mathrm{Pt} / \mathrm{Cr}$

(d) Lift-off process

Figure 5. Overview of fabrication steps for airflow meter sensor.

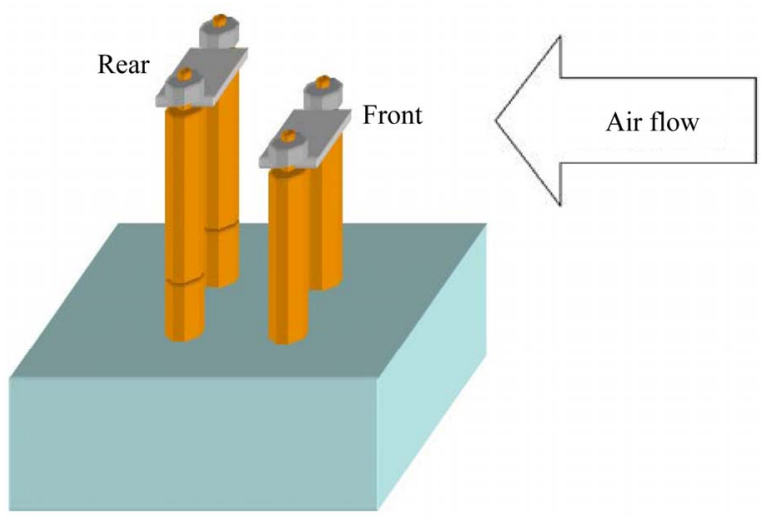

Figure 6. Schematic diagram of sensing mechanism.

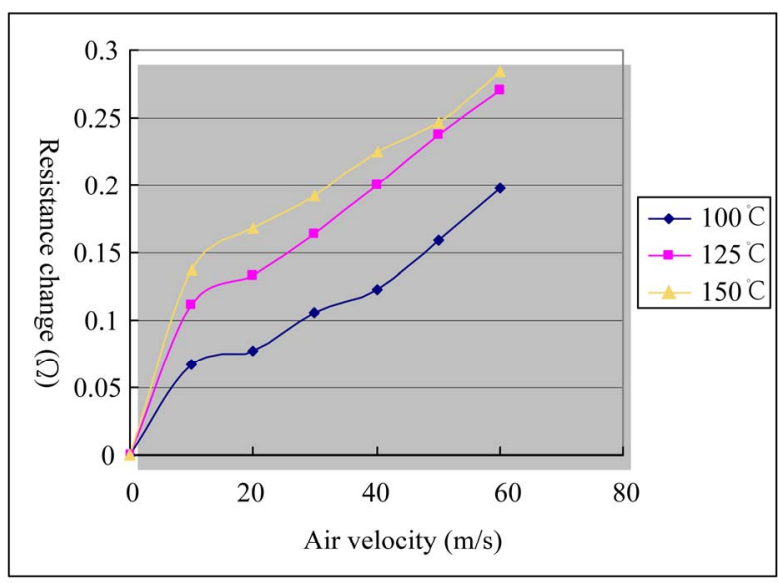

$$
\begin{aligned}
& 100 \mathrm{C} \rightarrow 0.0029 \Omega / \mathrm{ms}^{-1} \\
& 125^{\mathrm{C}} \rightarrow 0.004 \Omega / \mathrm{ms}^{-1} \\
& 150 \mathrm{C} \rightarrow 0.0041 \Omega / \mathrm{ms}^{-1}
\end{aligned}
$$

Figure 7. Front $30 \Omega$ heating, rear $40 \Omega$ sensing.

sor for airflow rate measurement applications. It can be clearly seen from Figures $\mathbf{7}$ and $\mathbf{8}$ that front sensing and rear heating yields the best performance. Figures 9 and 


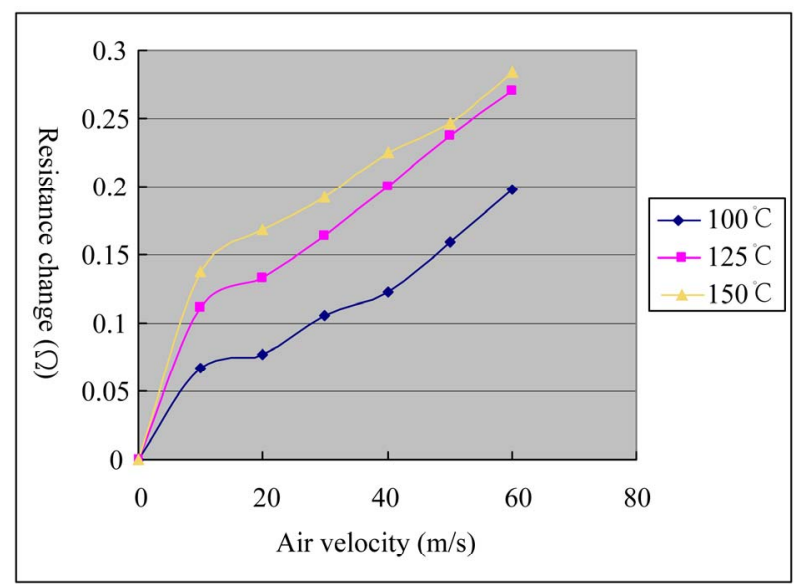

$\begin{aligned} 100 \mathrm{C} & \rightarrow 0.0038 \Omega / \mathrm{ms}^{-1} \\ 125^{\mathrm{C}} & \rightarrow 0.004 \Omega / \mathrm{ms}^{-1} \\ 150 \mathrm{C} & \rightarrow 0.0041 \Omega / \mathrm{ms}^{-1}\end{aligned}$

Figure 8. Front $30 \Omega$ sensing, rear $40 \Omega$ heating.

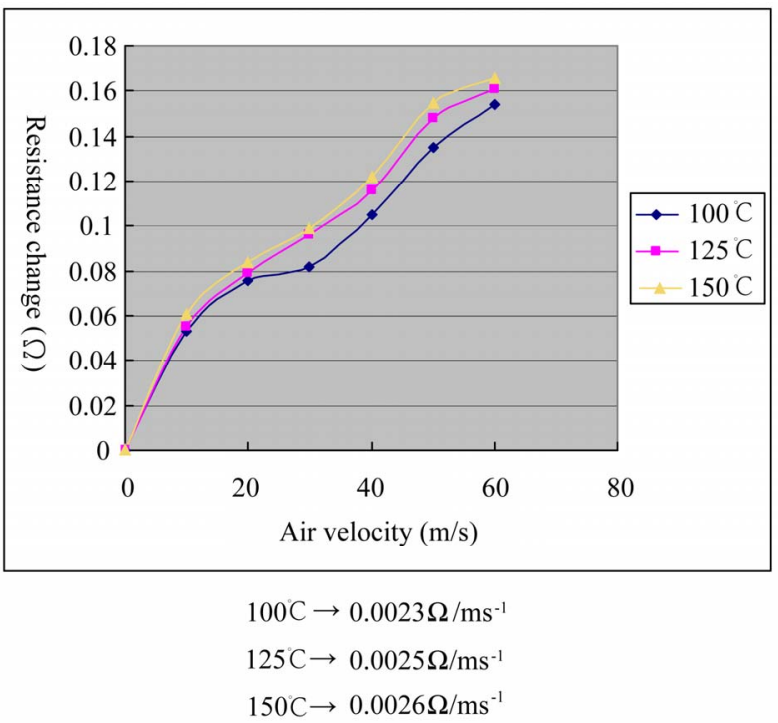

Figure 9. Front $20 \Omega$ sensing, rear $30 \Omega$ heating.

10 show the results of pairings of $20 \Omega$ and $30 \Omega$ and of $20 \Omega$ and $40 \Omega$, which yielded results similar to those of front sensing and rear heating. The single chips were then subjected to wind tunnel testing. Because it was known from testing the double chip that front sensing and rear heating yielded the best performance, the single chips were tested only in the front sensing and rear heating orientation. The results show the faster heat transfer and the faster response to the airflow rate. Curves were plotted of the relationship between the airflow anemometry and measured resistance (Figures 11-13). It can be found the response of the single-chip type of the flow sensors are faster due to their stronger heat conduction effect. The results reveal that the sensitivity increases as

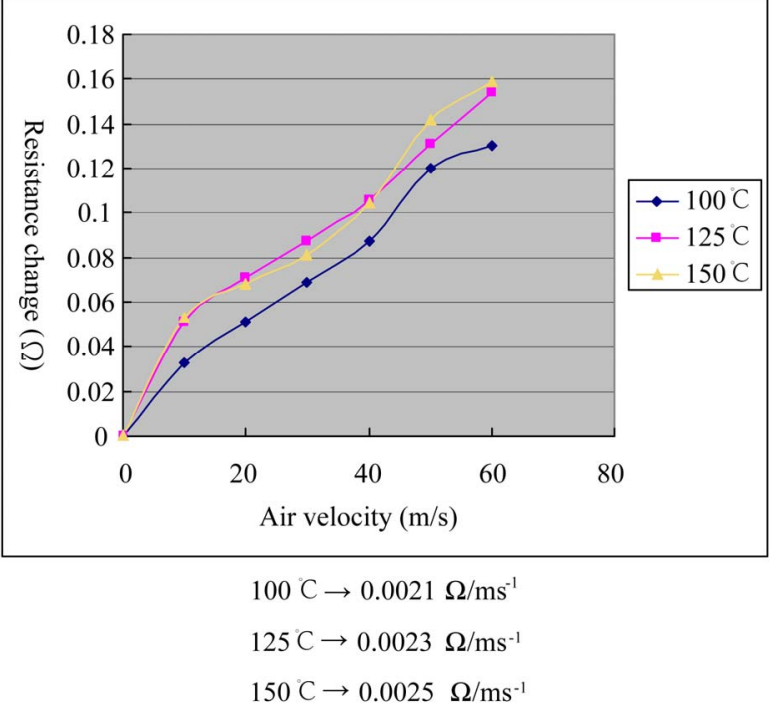

Figure 10. Front $20 \Omega$ sensing, rear $40 \Omega$ heating.

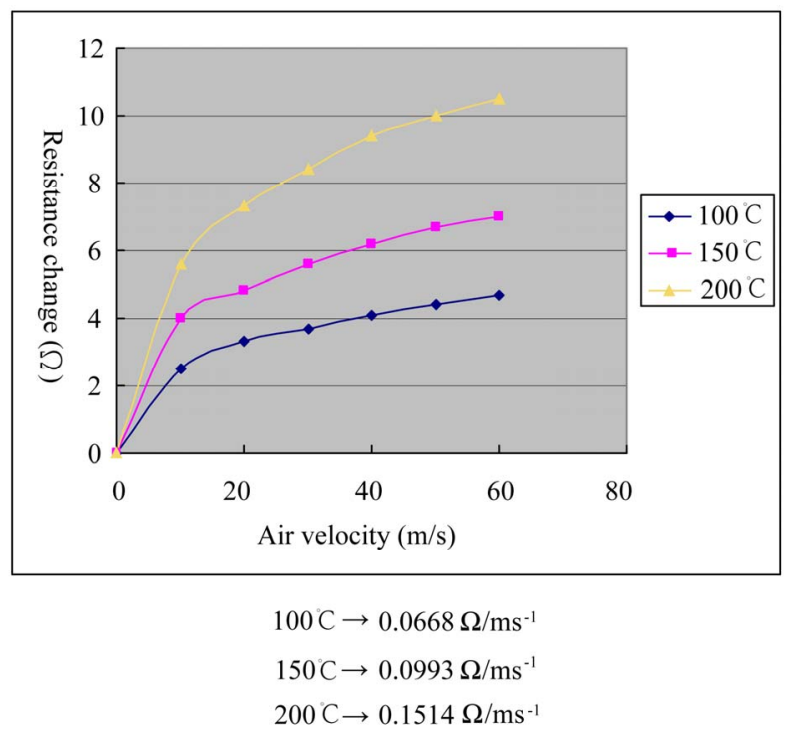

Figure 11. Front $40 \Omega$ sensing, rear $80 \Omega$ heating.

the resistance of the sensing element increases.

\section{Conclusions}

This study successfully demonstrated MEMS-based techlogy to produce an airflow anemometry sensor, and also varied parameters including resistance, size, direction, interval, and angle, and therefore deforms the piezoresistors patterned on their upper surfaces to observe the effect on sensing characteristics, and determine the best parameter values and greatest sensitivity. This work will aid the design of future car and motorcycle airflow anemometry meters. The double chip sensor was less sensitive than the single chip sensor, which indicates that dis- 


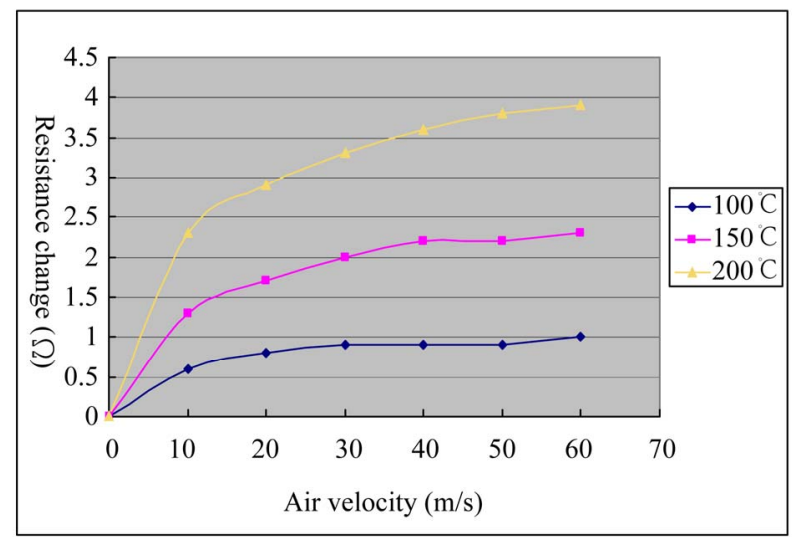

$$
\begin{aligned}
100 \mathrm{C} & \rightarrow 0.0132 \Omega / \mathrm{ms}^{-1} \\
150 \mathrm{C} & \rightarrow 0.0329 \Omega / \mathrm{ms}^{-1} \\
200 \mathrm{C} & \rightarrow 0.055 \Omega / \mathrm{ms}^{-1}
\end{aligned}
$$

Figure 12. Front $40 \Omega$ sensing, rear $120 \Omega$ heating.

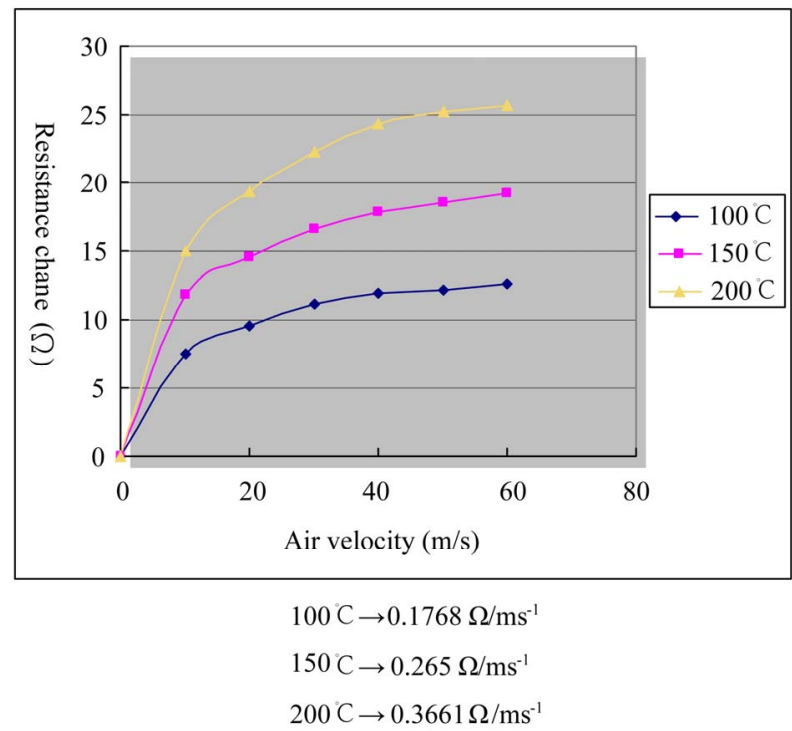

Figure 13. Front $120 \Omega$ sensing, rear $40 \Omega$ heating.

tance will influence sensitivity. The distance between the electrodes and sensing electrodes was found to be the main influencing factor, and the higher the environ- mental temperature, the better the performance.

\section{References}

[1] S. Kim, T. Nam and S. Park, "Measurement of Flow Direction and Velocity Using a Micromachined Flow," Sensors and Actuators A, Vol. 114, No. 2-3, 2004, pp. 312-318. doi:10.1016/j.sna.2003.12.019

[2] L. Qiu, S. Hein, E. Obermeier and A. Schubert, "Micro Gas-Flow Sensor with Integrated Heat Sink and Flow Guide," Sensors and Actuators A, Vol. 54, No. 1, 1996, pp. 547-551. doi:10.1016/S0924-4247(97)80012-X

[3] F. Mailly, A. Giani, R. Bonnot, P. Temple-Boyer, F. Pascal-Delannoy, A. Foucaran abd A. Boyer, "Anemometer with Hot Platinum Thin Film," Sensors and Actuators A, Vol. 94, No. 1-2, 2001, pp. 32-38. doi:10.1016/ S0924-4247(01)00668-9

[4] T. Neda, K. Nakmura and T. Takumi, "A Polysilicon Flow Sensor for Gas Flow Meters," Sensors and Actuators A, Vol. 54, No. 1, 1996, pp. 626-631. doi:10.1016/ S0924-4247(97)80027-1

[5] K. A. Makinwa and J. H Huijsing, "A Wind-Sensor Interface Using Thermal Sigma Delta Modulation Techniques," Sensors and Actuator A, Vol. 92, No. 1-3, 2001, pp. 280-285. doi:10.1016/S0924-4247(01)00584-2

[6] P. Bruschi, A. Diligenti, D. Navarrin and M. Pioto, “A Double Heater Integrated Gas Flow Sensor with Thermal Feedback," Sensors and Actuators A, Vol. 123-124, pp. 2005, pp. 210-215.

[7] C. Tropea, A. L. Yarin and J. F. Foss, "Springer Handbook of Experimental Fluid Mechanics," Springer, Berlin, 2007. doi:10.1007/978-3-540-30299-5

[8] R. Adamec, D. V. Thiel and P. Tanner, "MEMS Wind Direction Detection: From Design to Operation," Proceedings of 2nd IEEE Sensors Conference, Toronto, 2224 October 2003, pp. 340-343.

[9] K. A. A. Makinwa and J. H. Huijsing, "Constant Power Operation of a Two-Dimensional Flow Sensor Using a Thermal Sigma-Delta Modulation Technique," Proceedings of 18th IEEE Instrumentation and Measurement Technology Conference, Budapest, 21-23 May 2001, pp. 1577-1580. 